\title{
BIM AND GIS DATA INTEGRATION FOR THE EVALUATION OF BUILDING PERFORMANCE
}

\author{
N. Kadhim ${ }^{1}$ \\ ${ }^{1}$ Department of Civil Engineering, Diyala University, Baqubah, Diyala, Iraq, - nada.m.kadhim@uodiyala.edu.iq
}

Commission V, WG V/7

KEY WORDS: Building Performance, Post-Occupancy, CAD, BIM, ArcGIS, Structural Integrity, Spatial Analysis

\begin{abstract}
:
Building performance and condition assessment necessitate the integration of a variety of data sources, including building features, element/system properties, and supporting documentation. Previous research has concentrated on locating these data and establishing a building performance analytical framework using various techniques. However, due to challenges with traditional methods in monitoring the performance of a building within the default life of its construction, the maintenance procedure takes time and effort. This issue reflects the widespread omission of the appearance and progression of structural and non-structural faults in buildings, as well as a lack of methods for radical and speedy treatment. To overcome this issue, this research proposes a technique for integrating BIM and GIS data for building performance assessment. This will enable better-informed judgments about how to accelerate and optimise in-service asset operations and maintenance. The findings are also important for the advancement of GIS and BIM linked structural integrity evaluation solutions in the future. There are some important conclusions to be derived, as well as some possible future research subjects.
\end{abstract}

\section{INTRODUCTION}

Although buildings and other such structures are continuously shifting, these movements are generally so slight that they go unnoticed. Flaws, land subsidence, cornerstone collapse, degradation of the building fabric, and other factors can all contribute to this movement. These movements can lead to different types of cracks which can develop if a structure is unable to support this movement. For occupants, the sight of deformations and fissures can be uncomfortable and unsightly, and they might threaten the structure's stability, integrity, and sustainability if left unaddressed. Once cracking is identified, the reasons for it must be understood before a repair strategy is implemented. In this context, building information modelling (BIM) and geographic information systems (GIS) both play important roles in the lifecycle of construction and infrastructure projects. Thus, the integration of BIM and GIS technology is an important part of the overall solution for meeting the enormous construction and infrastructure development demands that will be required over the next 30 years and beyond.

\subsection{Related work}

The operation and maintenance processes of a building's lifecycle are as essential as its design and construction processes. Buildings deteriorate over time if they are not properly maintained. The lack of a maintenance strategy, combined with the natural ageing of the building, hastens the deterioration of existing structures (Kadhim, 2021). The prevention of flaws and the degradation of construction elements, as well as the extension of the service life of materials, necessitates the implementation of maintenance operations (Dobbs, Nitschke and Kendal, 2017). This can be achieved by conducting assessments of any damage and the general condition of a medium-rise building. Most infrastructure owners are eager to combine GIS and BIM data sources because procedures that potentially benefit from BIM and GIS technology are already in place in many situations. In this regard, efforts have been made by many researchers to integrate GIS and BIM data for the performance of different engineering purposes. For example, one study endeavoured to integrate GIS and BIM for indoor geovisual analytics (Wu and Zhang, 2016). In another contribution, the authors presented an automatic system for converting a BIM model's local coordinates to its real-world geographic coordinates (Diakite and Zlatanova, 2020). A novel framework for importing laserscanning data into BIM using Autodesk Revit to a 3D building model has also been created, for the purposes of structural building analysis (Kadhim, Mhmood and Abd-Ulabbas, 2021). Another recent study considered a building condition risk assessment model integrated into BIM using a data model (Alavi, Bortolini and Forcada, 2022). Despite the integration of BIM and GIS information receiving attention in recent years regarding a wide range of issues, there is still a clear lack of practical evaluation of the analysis of the actual performance of a building in terms of its condition and structural integrity.

\subsection{Motivation and contribution}

As a system of record for its geospatial information, ArcGIS data can be arranged in geodatabases. BIM content can be represented in a geodatabase, providing access to all the database's features. Examples of these capabilities are maintenance, connecting buildings to other document management systems, leveraging relationship classes, and increasing visualisation by conserving fine details from BIM files. Individual disciplines from various Revit files can be merged into one building using a geodatabase, and multiple buildings can be combined into a single campus. Individual buildings have distinct names, allowing searches for assets within a given building or group of buildings. The properties or 
geometry of the buildings can also be maintained and altered after being saved in a geodatabase. In a building dataset, 3D assets from BIM files are kept in multipatch feature classes. This combining of the merits of ArcGIS and BIM data can be exploited to improve understanding and take a comprehensive view of structural defects, such as cracks, in a specific building. Interacting with CAD/BIM data and drawings in this area could permit the handling of extremely detailed building models based on consistent and clear data formats, as well as help identify the positions of the defects and assessment of the extent of damage and its influence on the building. In this context, the aim of this paper has two objectives: to integrate the two technologies for evaluating an in-service building performance, and to exploit this integration for analysing and evaluating the building's real performance in terms of its condition and structural integrity.

The remainder of the paper is organised as follows. Section 2 delves into the specifics of our method. The findings of our experiments for a specific campus building are presented in Section 3, and Section 4 concludes with the discussion.

\section{METHOD}

Multiple integral processes compensate our proposed approach. Pre-processing is the first stage in preparing the datasets for analysis. Then, these datasets are used in initial symbology by creating a heat map, conversion tools (feature class to feature class and feature to polygon), spatial analyst tools (kernel density and zonal statistics as a table, based on the former results), data management tools (add join to classify the magnitude of every crack). Lastly, the 3D BIM model of a building which was created with its Revit files is georeferenced. The most serious impact of the building's cracks is then visualised according to the analysis results on the 3D building model's walls. The complete flowchart for our strategy is shown in Figure 1.

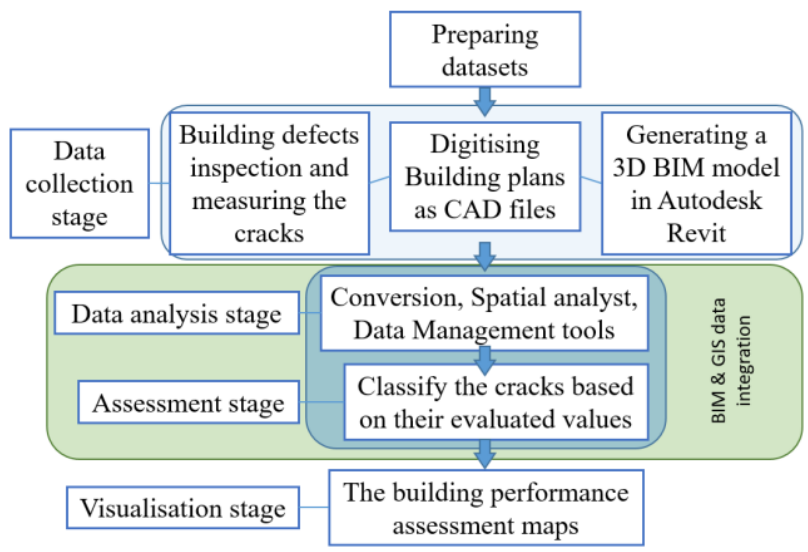

Figure 1. A brief review of the suggested workflow of the presented approach

\subsection{Datasets}

A campus building, the Civil Engineering Department, was selected. This medium-rise two-storey building was built by a German construction company in 1976. Although there has been restoration from time to time for this building, as an actual structural assessment of the building's defects as a result of its long-term operation has not yet been completed. The building's base drawings were redrawn to produce a digital version of the AutoCAD drawings. Afterwards, a three-dimensional model of the building was produced using Autodesk Rivet software, based on the produced AutoCAD drawings. Both were used to assess the condition of the current building.

\subsection{Crack position inspection and measurement}

Cracks which emerge on various concrete parts, such as beams, columns, bases, and load-bearing walls, are the most problematic since they can indicate a differential decrease in the bases or insufficient reinforcement, as well as the concrete's incapacity to take loads. Due to problems in a building's foundations, such as faulty foundation soil, substantial subsidence, and displacement in beams and roofs above and below walls, wide diagonal fissures can occur on the borders of windows and doors. Therefore, we examined the position of each crack in the building's structure, including on every external wall of every room of the two floors, and the external walls of the building. Thereafter, the width, length, direction and inclination angle for each crack was measured using the concrete crack measuring microscope. We classified the cracks depending on their visual inspection and measurements, as in Table 1.

\begin{tabular}{|c|c|}
\hline Crack width range & Crack denotation \\
\hline$<0.05 \mathrm{~mm}$ & Very small \\
\hline $0.05-0.2 \mathrm{~mm}$ & Small \\
\hline $0.2-0.5 \mathrm{~mm}$ & Large \\
\hline$>0.5 \mathrm{~mm}$ & Very large \\
\hline
\end{tabular}

Table 1. Crack classification based on measurements and visual inspection

\subsection{Building cracks assessment}

The AutoCAD files (2D) and Revit model files (3D) of the selected building were added and integrated as geospatial data into ArcGIS content, after geolocating and georeferenced the $2 \mathrm{D}$ building plans the 3D Revit model to a real-world location. This step was accomplished by assigning a coordinate system in ArcGIS Pro and adjusting the position of data. To estimate the severity of the crack on the building structure and the effect of this crack on its neighbouring building elements, we employed the kernel density tool. This tool enables the calculation of a magnitude-per-unit area from point features using a kernel function to fit a smoothly tapered surface to each point. The cracks were represented and projected on their real location as a point feature class within the ArcGIS Pro 2.8 environment. Next, we summarised the values in each zone/area as a table by employed zonal statistics as table tool, and then we joined the two attribute tables from the two processes to determine the most harmful side of the building due to these cracks. We calculated the percentile of all pixels in the valuation raster that were in the same zone as the outcome cell. In the final step, the findings of the spatial analysis processes were classified by the bivariate colours symbology method to attain the final maps of the entire building. The two essential parameters in the assessment process were crack width and the mean of the impact zone of these cracks. To present the 3D model of the selected building, we added the model to the scene ArcGIS environment to identify the location of the cracks most harmful to the construction in its $3 \mathrm{D}$ form. 


\section{RESULTS}

The analysis of the results illustrated the ability to deliver new benefits from connecting GIS and BIM. In particular, the structural integrity assessment (SIA) of existing buildings in our presented novel approach can determine if a structure is capable of safely and reliably enduring service conditions during its expected lifetime. Figures 2 and 3 show the georeferenced building on its exact actual location and its true UTM coordinates system.
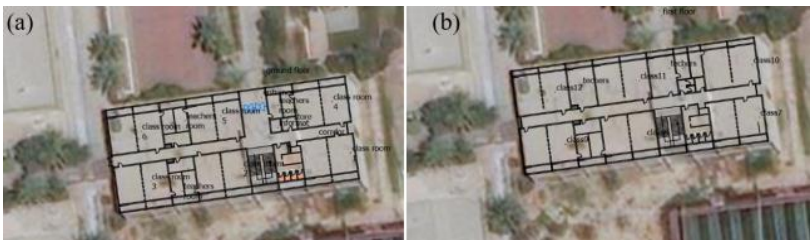

Figure 2. The projected 2D plans: (a) the ground floor and (b) the $1^{\text {st }}$ floor of the building

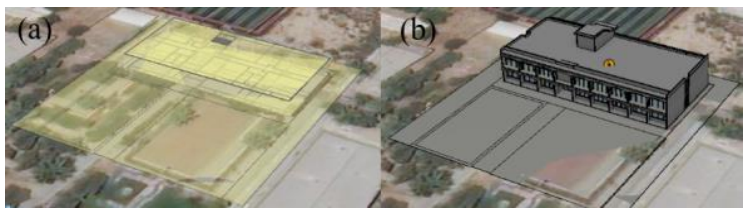

Figure 3. The projected 3D model: (a) the georeferencing process (b) the geolocating all related files of the Revit model

The results from the process of projecting the cracks as points on different parts of the construction are shown in Figure 4. The initial assessment of each crack type, and the severity of a crack's deterioration, were derived by applying the heat map technique to every crack's location. This provides us with a significant indication of how dangerous each crack is in the building.

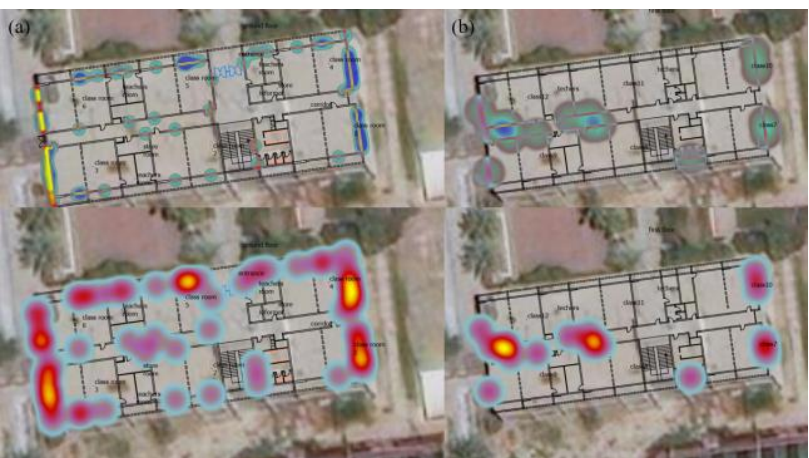

Figure 4. The heat maps containing the points: (a) the ground floor (b) the $1^{\text {st }}$ floor of the building

Point features are drawn as a dynamic, representational surface of relative density in heat map symbology. Because there were many dots (cracks) close together, making them harder to identify, we symbolised the kernel density map into the 7 classes with the search radius from 1 to 2 map units, as in Figure 5. The classification maps of the point features (the building cracks) were extracted depending on the cracks attribute table, derived from the spatial analysis process, as shown in Figures 6 and 7.

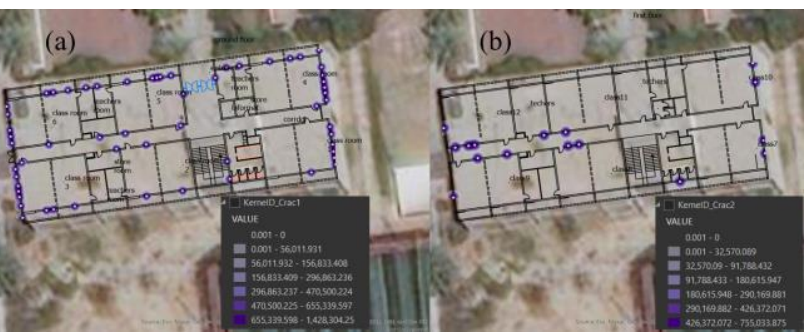

Figure 5. Kernel density of the building cracks based on the area rate of crack width: (a) the ground floor and (b) the $1^{\text {st }}$ floor of the building

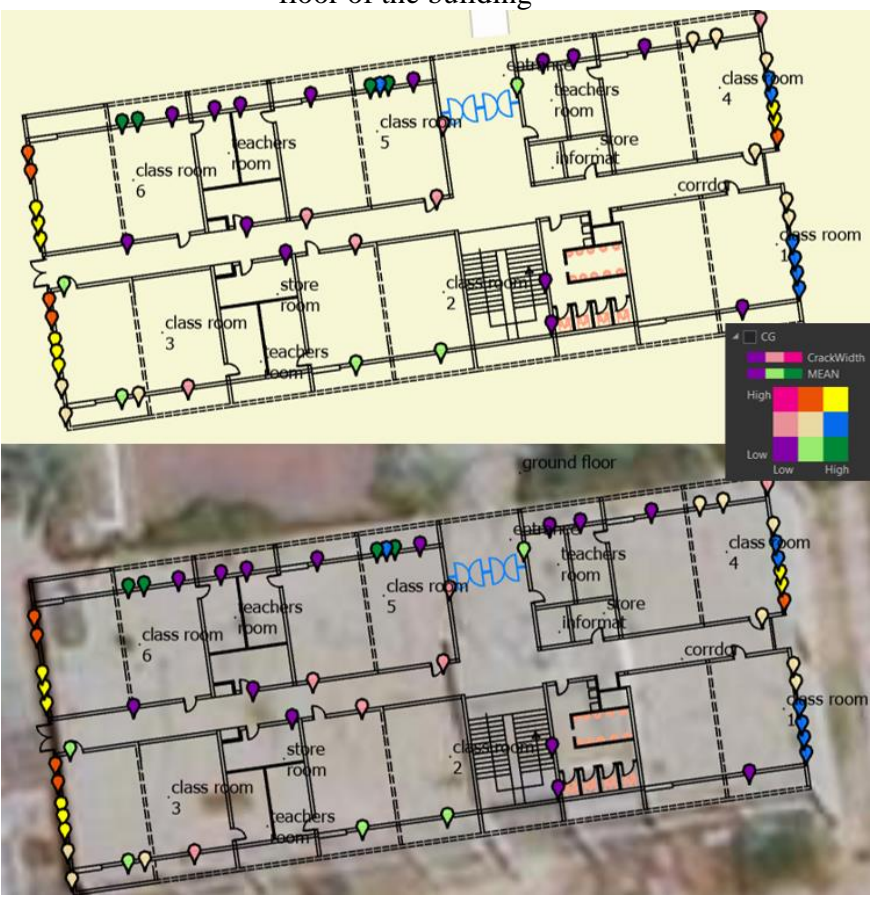

Figure 6. The ground floor of the building with the classified cracks

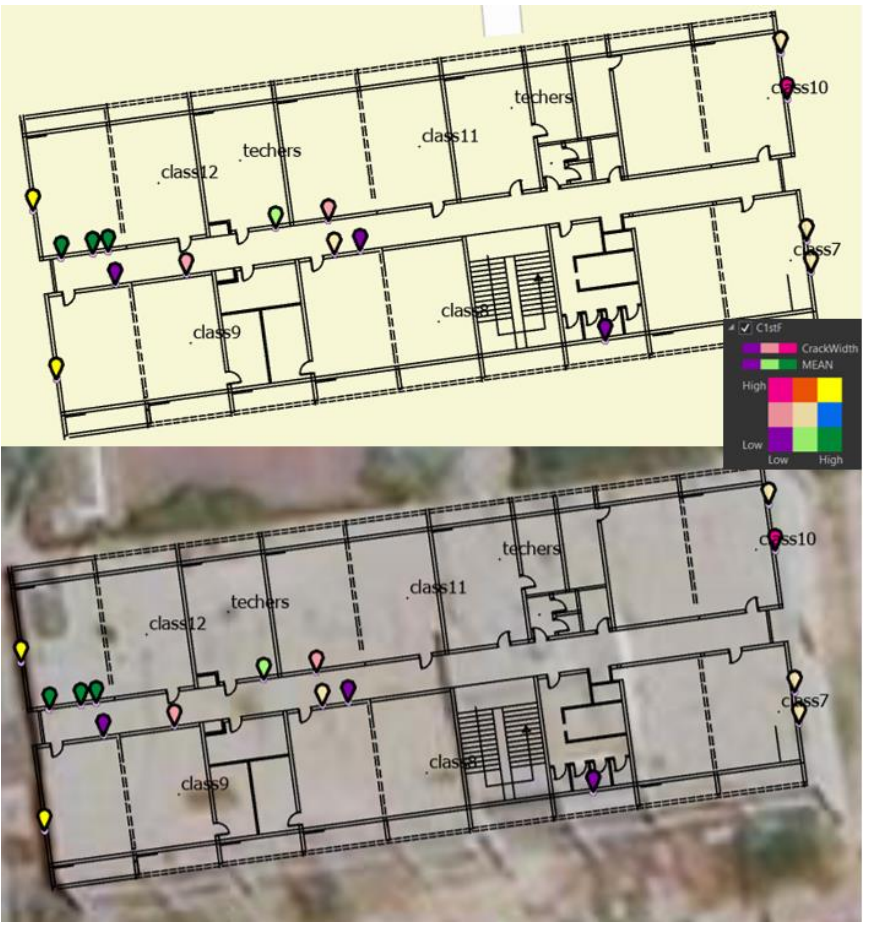

Figure 7 The $1^{\text {st }}$ floor of the building with the classified cracks 


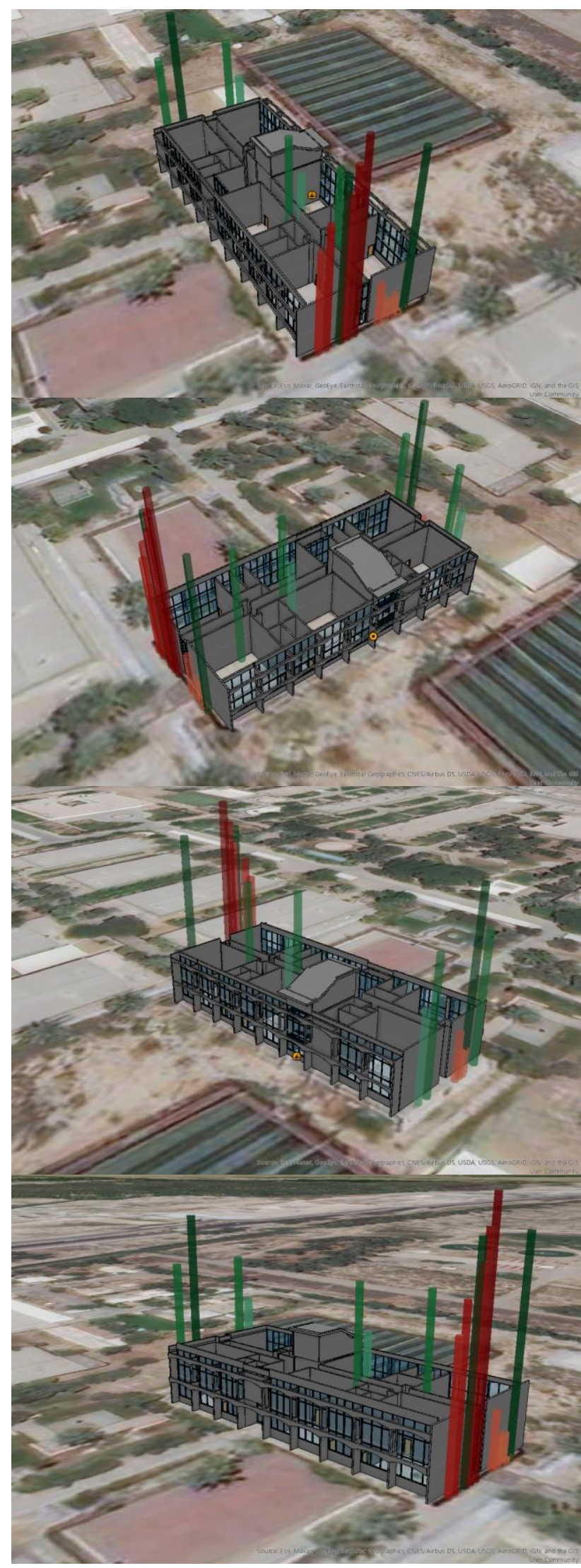

Figure 8. The 3D BIM model of the building with the classified cracks from different side views

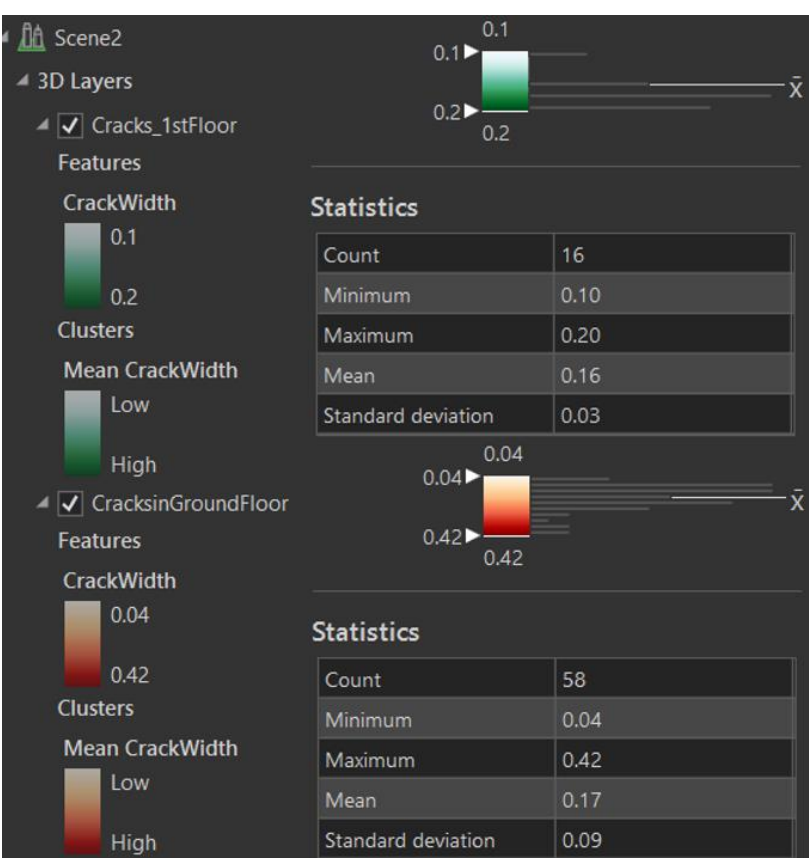

Figure 9. The statistical values of the extrusion of the building cracks and the aggregation range of the cracks (the point features) into dynamic point clusters

In Figure 8, the classified point's crack values were correctly projected on the 3D BIM model of the building. However, we extruded these values to improve the view for presentation proposes. Two colour schemes were employed to differentiate the cracks' locations between the two floors. The red range is for the cracks on the ground floor and green represents the cracks on the first floor. The statistical values of the visualisation process applied to the 3D BIM model is shown in Figure 9.

\section{DISCUSSION AND CONCLUSION}

The study shows that by combining BIM and ArcGIS data, a robust technique can provide a quick and accurate assessment of a building's condition and performance. However, certain aspects were not covered in the experimental work which need to be discussed further. The building cracks were found to be mostly tensile and oriented approximately parallel to the direction of highest compressive stress, according to the results of the examination. A shear crack's starting point is sometimes defined as the point where the maximum primary stress (compressive stress) direction is tangent to the fault edge. On the other hand, the results of the spatial density and statistical analyses are in reasonable agreement with the field data. Furthermore, displaying cracks on a 3D model depicts the most influential side of the building walls. This will make future treatment of these cracks for building maintenance much easier. However, the findings suggest that the building's condition is still good, and we may continue to use it as a campus facility. Nevertheless, some cracks are significant and, if left untreated, may become even more hazardous. An appropriate technical solution should thus be regularly implemented. Our novel method capitalises on existing developments in BIM and GIS software to increase improvements in assessment results. This can lead to better-informed decisions to accelerate and improve the operations and maintenance of in-service assets. The results are also of significance to facilitate future development of GIS and BIM integrated solutions in SIA. In future work, we can 
improve certain features of our strategy. Since real cracks should be realistically represented on the 3D building model, there is a need to develop an automatic technique or tool to present the actual cracks and defects of any building, in particular on the 3D BIM model. Furthermore, because BIM models are not designed for use in GIS contexts, more research into their integration in terms of data modelling is needed.

\section{ACKNOWLEDGEMENTS}

The author appreciate all of the reviewers' suggestions for enhancing this work. I also would like Mr Mohammed Salih and Mrs Amira for their support and useful discussion.

\section{REFERENCES}

Alavi, H., Bortolini, R. and Forcada, N., 2022. BIM-based decision support for building condition assessment, Automation in Construction, 135(November 2021), p. 104117. doi: 10.1016/j.autcon.2021.104117.

Diakite, A. A. and Zlatanova, S., 2020. Automatic georeferencing of BIM in GIS environments using building footprints, Computers, Environment and Urban Systems, 80(December 2019), p. 101453. doi: 10.1016/j.compenvurbsys.2019.101453.

Dobbs, C., Nitschke, C. and Kendal, D., 2017. Assessing the drivers shaping global patterns of urban vegetation landscape structure, Science of the Total Environment, 592, pp. 171-177. doi: 10.1016/j.scitotenv.2017.03.058.

Kadhim, N., Mhmood, A. D. and Abd-Ulabbas, A. H., 2021. The creation of 3D building models using laser-scanning data for BIM modelling, IOP Conference Series: Materials Science and Engineering, 1105(1), p. 012101. doi: 10.1088/1757899X/1105/1/012101.

Kadhim, N.M.S.M., Mohammed, A.A. and Kadhim, N., 2021. THE ANALYSIS OF PANDEMIC EFFECTS ON DESIGN THINKING FOR ARCHITECTS. Design Engineering, pp.7264-7282.

Wu, B. and Zhang, S., 2016. Integration of GIS And BIM for indoor geovisual analytics, International Archives of the Photogrammetry, Remote Sensing and Spatial Information Sciences - ISPRS Archives, 41(July), pp. 455-458. doi: 10.5194/isprsarchives-XLI-B2-455-2016. 